\title{
Effect of Ulceration on the Therapeutic Benefit of Surgery in Patients with Stage IV Melanoma: A Surveillance, Epidemiology, and End Results Analysis from 2004-2015
}

\section{Qiqi Zhao}

The second hospital of Dalian Medical University https://orcid.org/0000-0003-0001-2477

\section{Zeyun Gao}

Department of occupational and enviromental health,Dalian Medical University

Xuezhu Xu ( $\nabla$ xxz@dmu.edu.cn )

The second hospital of Dalian Medical University

\section{Research}

Keywords: Stage IV, Skin Malignant Melanoma, Surgery, Prognosis, SEER

Posted Date: October 26th, 2020

DOI: https://doi.org/10.21203/rs.3.rs-96111/v1

License: (c) (1) This work is licensed under a Creative Commons Attribution 4.0 International License.

Read Full License 
1 Effect of Ulceration on the Therapeutic Benefit of Surgery in Patients with Stage IV

2 Melanoma: A Surveillance, Epidemiology, and End Results Analysis from 2004-2015

3 Qiqi Zhao ${ }^{1 \#}$, Zeyun $\mathrm{Gao}^{2 \#}$, Xuezhu $\mathrm{Xu}^{1 *}$

4

5 1.Department of Dermatology, The Second Affiliated Hospital of Dalian Medical University,

6 Dalian, Liaoning, People's Republic of China

7 2.Department of Occupational and Environmental Health, Dalian Medical University, No. 9

8 W. Lvshun South Road, Dalian 116044, China

$9 \quad *$ Corresponding author: Xuezhu Xu

10 Department of Dermatology, The Second Affiliated Hospital of Dalian Medical University,

11 Dalian, Liaoning, People's Republic of China.

12 Email: xxz@dmu.edu.cn

13 \#Both authors contributed equally to this work.

14

15

16

17

18

19

20

21

22

23 
Abstract:

27 Background: The effects of various surgical options and ulcerations on the survival of patients with stage IV skin malignant melanoma are unknown. Therefore, we evaluated the potential of these factors as prognostic markers in patients with stage IV malignant melanoma.

Methods: We included 5760 patients from 2004-2015 who are screened from the SEER datasets in the study. The patients were divided into four groups: the $\mathrm{R}_{0}$ group, the primary tumor resection group, the metastasectomy group, and the no-resection group. The median follow-up survival time and overall survival were compared between the four groups as primary outcomes.

Result: The $\mathrm{R}_{0}$, primary tumor resection, metastasectomy, and no-resection groups had median survival times of $11,13,20$, and 4 months, respectively $(p<0.001)$. Cox (proportional hazards) regression models estimated that patients in the $\mathrm{R}_{0}$, primary tumor resection, and metastasectomy groups had longer survival benefits, with hazard ratios of $0.396(95 \%$ confidence interval [CI], 0.347-0.453), 0.509 (95\% CI, 0.465-0.556), and 0.481 (95\% CI, 0.447-0.519), respectively.

Conclusion: We highlight the importance of surgery in metastatic melanoma; each surgical group in this study is independently correlated with increased survival. In addition, the patient's ulceration status is able to predict surgical treatment; however, in the ulcerated melanoma cases, caution should be exercised when considering a metastasectomy.

Keywords: Stage IV, Skin Malignant Melanoma, Surgery, Prognosis, SEER

\section{Background}

50 The common and increased morbidity tumor is represented by melanoma, which is among the

51 potentially lethal cancers. Currently, more than 1.2 million Americans are living with the

52 disease ${ }^{1-3}$. Approximately $20-30 \%$ of stage IV melanoma patients initially diagnosed with

53 locoregional disease will develop distant metastases ${ }^{4,5}$, and $8 \%$ will present with concurrent 
regional and distant metastases ${ }^{6}$. A study on stage IV skin malignant melanoma patients demonstrated the 1-year survival rate to be $33-62 \%^{7}$. Ultimately, patients with metastatic melanoma have poor prognoses.

Several retrospective clinical studies have evaluated the survival of patients with stage IV malignant melanoma undergoing tumor resection ${ }^{8}$. Surgical treatment is essentially a suitable standard of care ${ }^{9,10}$. There is great clinical significance in evaluating the available surgical treatments for patients with metastatic melanoma based on the pathological criteria such as ulceration. Ulceration is an important independent hallmark of melanoma recurrence and adverse survival $^{5}$. To the best of our knowledge, the existing literature lacks informative analyses regarding the prognostic factors associated with survival in patients with different surgical interventions. Furthermore, the effect of ulceration on decisions regarding different resection sites in this stage IV skin malignant melanoma population is poorly evaluated. Therefore, this study aimed to assess the significance of surgical treatment choice and ulceration status as predictive markers by conducting a retrospective population-based study of stage IV melanoma patients.

\section{Materials and methods}

\subsection{Data source}

The Surveillance, Epidemiology, and End Results (SEER) population-based program, supported by the National Cancer Institute, is one of the most representative sources of oncology statistics in the USA. SEER cases are collected from 18 accredited public population-based cancer registries, accounting for roughly $28 \%$ of the annual cancer diagnoses of the USA. This is comparable to the overall characteristics of the general population. We screened the cases using the statistical SEER*Stat software (version 8.3.6.1) in the client-server mode. Approval from the Institutional Research Board (IRB) was waived due to the data being anonymous and freely accessible. The data were extracted, interpreted, and analyzed on the basis of the SEER guidelines. 
83 We identified 5760 eligible patients that were initially diagnosed with stage IV malignant melanoma using the International Classification of Disease for Oncology, 3rd Edition. We collected the patients' detailed clinicopathological characteristic data (Figure 1). We separated the patients into four groups according to surgical treatment: the $\mathrm{R}_{0}$ group (resection of all malignant lesions), the primary tumor resection group, the metastasectomy group, and the no-resection group. To assess the clinicopathological predictors for different melanoma-directed surgical interventions, patients were subdivided into categories based on their ulceration status.

\subsection{Statistical analysis}

The balance of categorical covariates in the patient characteristics between the four groups was evaluated using the Pearson's Chi-square $\left(\chi^{2}\right)$ test. We used the log-rank test to compare the survival distributions in the resection cohorts. We constructed Kaplan-Meier curves to estimate and compare the overall survival (OS), melanoma-specific survival (MSS), and median survival time (MST) between the four groups. We also compared the MST and OS of a stratified analysis according to the pathological melanoma ulceration status between the subgroups. We report the significant odds ratios (OR) with a 95\% confidence interval (CI). We assessed survival factors utilizing the Cox proportional hazards regression. We used multivariable analyses (logistic regression) to find the baseline parameters meaningfully associated with different surgical interventions. Significant hazard ratios (HR) with 95\% CI are reported. For all tests, the level of statistical significance was expressed as a $p$-value $<0.001$. SPSS software (version 22, Chicago, Illinois) was used to perform all statistical analyses.

\section{Results}

\subsection{Demographic features and clinical and pathological data}


The clinicopathological parameters and demographic data are presented in Table 1. We

110 analyzed the data of 5760 patients with metastatic cancer concurrent with a primary tumor;

111 among 5760 patients, 1297 (22.5\%) patients underwent surgery of the primary tumor only,

112 and 1447 (25.1\%) patients underwent a metastasectomy only. Resections of the primary and

113 metastatic sites were performed in 375 (6.5\%) patients; $2641(45.9 \%)$ patients did not

114 undergo any surgical treatment. The ulcerated melanoma cases comprised $16.2 \%$ of the total

115 cases. Significant differences were found in the age at diagnosis, primary site, disease spread,

116 and ulceration status. Features of the ulceration subgroups are summarized in Table S1.

\section{$118 \quad 3.2$ Survival outcome}

119 Among the patients, 4492 (78.0\%) melanoma-specific deaths were identified. The median OS 120 and MSS of the entire cohort were 7 months (95\% CI: 6.6-7.4 months, 5-year OS=6.5\%) and 1218 months (95\% CI: 7.6-8.4 months, 5-year OS=8.4\%), respectively (Table S2). The KaplanMeier survival analysis curves (Figure 2) estimated the $\mathrm{R}_{0}$ group (MST=20 months, 5-year

$123 \mathrm{OS}=28.8 \%, p<0.001)$ have a longer survival outcome than the primary tumor resection group

124 (MST=11 months, 5-year OS=18.5\%, $p<0.001)$ and the no-resection group (MST=4 months, 5 -year OS=6.5\%, $p<0.001)$. There were numerical differences in the OS and MSS between the primary site resection group and the metastasectomy group, but none were significant. The metastasectomy group and the $\mathrm{R}_{0}$ group showed similar results.

\subsection{Ulceration makes predictions from surgery to outcome}

130 Table 2 shows the MST duration and OS at three years of the subgroups stratified according

131 to ulceration status. The stratified Kaplan-Meier survival curves are presented in Figure 3.

132 In the non-ulcerated melanoma subgroup, the $\mathrm{R}_{0}$ resection group (MST=25 months, 95\% CI:

133 14.1-35.9 months, $p<0.001$ ), primary excision group (MST=13 months, 95\% CI: 10.5-15.4

134 months, $p<0.001$ ), and metastasectomy group (MST=16 months, 95\% CI: 11.5-20.5 months, $135 p<0.001)$ showed distinctly longer survival times compared to the no-resection group 136 (MST=4 months, 95\% CI: 3.3-4.7 months, $p<0.001$ ). Furthermore, differences in survival 
137 time failed to reach significance between the $\mathrm{R}_{0}$ group and the primary site excision group

138 ( $p=0.012$ ) or between the $\mathrm{R}_{0}$ group and metastasectomy group ( $\left.p=0.069\right)$. No additional

139 survival benefit was detected when the metastasectomy group was compared with the

140 primary excision group $(p=0.447)$.

141 In the ulcerated melanoma subgroup, survival time was higher after receiving $\mathrm{R}_{0}$ therapy than

142 after receiving a primary site resection $(p<0.001)$. Furthermore, both the $\mathrm{R}_{0}$ group (MST=13

143 months, 95\% CI: 9.9-16.1 months, $p<0.001)$ and primary site excision group (MST=9

144 months, 95\% CI: 7.9-10.1 months, $p<0.001)$ showed an improved OS when compared with

145 the no-resection group (MST=4 months, 95\% CI: 2.6-5.4 months, $p<0.001$ ), but not with the

146 metastasectomy group (MST=7 months, 95\% CI: 4.1-9.9 months, $p=0.049$ ).

\section{$148 \quad 3.4$ Relevance of clinicopathological parameters and OS}

149 The Cox (proportional hazards) multivariate analysis results are presented in Table 3.

150 Predictive factors of poor OS were identified as age over 50 years, higher lactate

151 dehydrogenase levels, type of surgical treatment, ulceration, and metastases in the brain,

152 liver, lungs, or bone. Out of the four groups, the $\mathrm{R}_{0}$ group had the best HR of 0.396 (95\% CI,

153 0.347-0.453). Patients with primary site resection and metastasectomy showed significantly

154 favorable survival and outcome benefits, with HRs of 0.509 (95\% CI, 0.465-0.556) and

1550.481 (95\% CI, 0.447-0.519), respectively.

\subsection{Predictors for surgical intervention}

158 To assess the relationship between ulceration and surgery, extracted cohorts were further 159 subdivided into the no-resection group and the surgery group (all patients who underwent 160 surgical interventions). The univariate logistic regression analysis (Table 4) revealed that 161 ulceration was a significant independent prognostic factor of surgery $(\mathrm{OR}=0.236,95 \% \mathrm{CI}$ :

$162 \quad 0.189-0.295, p<0.001)$.

\section{Discussion}


165 After a median follow-up of four months, the risk of melanoma-specific deaths in the noresection group was significantly higher than that in the surgical group. The multivariate Cox model showed that the three surgical groups (resection of all malignant lesions, primary site resection, and metastasectomy) were independently associated with increased survival. Despite improvements in survival, largely attributed to the new landscape of multidisciplinary effective systemic therapies over the last decade ${ }^{11}$, surgical resection remains the only option that improves survival by reducing the tumor burden and interrupting the metastatic cascade. Therefore, surgical resection has an ongoing and expanding role in systemic medical treatment ${ }^{11-13}$. Early and accurate assessments of surgical treatment patterns are necessary for proper management, and for ensuring that surgical resection is utilized to its full potential.

176 The strategy of complete surgical resection in appropriately screened patients is supported by several clinical trials ${ }^{14,15}$. In a retrospective analysis of patients in the Multicenter Selective Lymphadenectomy Trial, patients with stage IV melanoma who underwent wide-scale surgical resection had longer disease-free survival outcomes than those who received systemic medical therapy alone ${ }^{3}$. In a large prospective trial, stage IV melanoma patients who underwent complete resection had a better median OS than patients who underwent nonsurgical treatment ${ }^{16}$. Additionally, in a population-based meta-study, the survival outcomes (OS and MSS) were improved in patients who underwent primary tumor resection 12 .

185 A complete metastasectomy was historically a potentially effective treatment option in 186 advanced metastatic cases involving the skin and visceral organs ${ }^{17}$. Patients with stage IV melanoma who underwent metastasectomies had better OS than those who did not ${ }^{6}$. A metastasectomy is recommended for patients with metastatic melanoma by the National

189 Comprehensive Cancer Network guidelines ${ }^{18}$; however, the guidelines lack proper 190 descriptions regarding whether primary tumor resections should be routinely practiced in 191 advanced melanoma patients or not, and the conclusions are limited by conditions such as 
192 solitary lesions. Selection bias may be contributing to observed associations between survival

193 benefit and surgery in previous retrospective studies $6,8,11,19,20$.

194 In this study, patients with stage IV malignant melanoma who underwent surgery showed an

195 improved survival compared with those who did not undergo surgery. This study also showed

196 that survival outcomes were better among the surgical groups than among the no-resection

197 group in the non-ulcerated subgroup. However, when comparing the three surgery groups

198 with each other, no additional survival benefits were found. Within the ulcerated subgroup,

199 the $\mathrm{R}_{0}$ group had better survival benefits than the primary site excision group. There were

200 similar outcomes for the metastasectomy and no-resection groups.

201 Ulceration of cutaneous melanoma is defined as defective layers of the epidermis overlying

202 any portion of the primary melanoma, with an associated host response based on the

203 histopathological microscopic examination ${ }^{21,22}$. Studies are increasingly reporting a trend of

204 a remarkably similar MSS between melanoma patients with ulceration and primary tumor

205 patients having the greatest thickness but without ulceration leading to the American Joint

206 Committee on Cancer melanoma T staging criteria ${ }^{18,23,24}$. MSS in a wide range is influenced

207 by the T stage during clinically early-stage melanoma. A detailed analysis of 521 ulcerated

208 and 4140 non-ulcerated patients revealed that microscopic ulcerations have an independent

209 effect on MSS in primary melanoma (27). The risk of dermal invasion and development of

210 metastasis in the brain and other regions is estimated on the basis of histopathological

211 heterogeneous phenomenon such as ulceration ${ }^{20,25}$; however, convincing explanations

212 regarding how ulceration mechanisms hold prognostic value have been poorly presented.

213 Ulceration results from the progressive loss of epithelium due to the intercellular bonds of the

214 epithelium weakening; therefore, ulceration is progressive and includes ongoing alterations

215 that increase the number of inflammatory cells, proliferation of tumor cells, tumor growth,

216 and increases the risk for metastases. One study reported that ulceration was the only primary

217 tumor characteristic that remained an independent survival predictor on multivariate analysis

218 after brain metastasis ${ }^{25}$. However, we suggest that the choice to pursue resection in stage IV

219 melanoma patients provides a dilemma. For example, the most important objective is 
complete removal of the tumor; patients with ulceration are more likely to undergo resection,

221 beyond just for survival benefit. The staging of patients with distant metastases is currently dictated by the site of metastases and no longer by the primary tumor characteristics ${ }^{23}$. The influence of ulceration (or lack thereof) in the primary tumor of patients with American Joint

224 Committee on Cancer stage IV melanoma has not been specifically defined. Our results may help address this knowledge gap with support from further prospective findings or trials. This study has several limitations. First, the inclusion of patients eligible for surgery introduced an inherent granular data bias; the most common resections, relation to OS expectancy, and high selection of patients eligible for complete $\mathrm{R}_{0}$ resection of all metastatic cutaneous, subcutaneous, and visceral malignant lesions were not taken into account. Second, information on micrometastases, tumor dormancy effects, and use of systemic regimens were not evaluated. Third, detailed complications, adverse events, and clinical considerations are not available in the SEER database.

\section{Conclusion}

235 The increasing efficacy of systemic treatment algorithms provides new treatment

236 opportunities; however, surgery still plays a major role in stage IV melanoma treatment. The ulceration in the primary tumor is one of the predictive factors of poor OS in patients with stage IV melanoma. Despite its limitations, our population-based study still found that both the OS and MSS of patients could benefit from each of the three surgical types ( $\mathrm{R}_{0}$ resection, primary excision, and metastasectomy). To conclude, patients with ulceration might predictably benefit from surgical treatment; however, caution should be exercised when considering metastasectomy in patients with ulcerated melanoma.

\section{List of abbreviations} overall survival (OS)

247 melanoma-specific survival (MSS)

248 median survival time (MST)

249 odds ratios (OR) 
confidence interval (CI)

251 hazard ratios (HR)

\section{Declarations}

\section{Ethics approval and consent to participate}

255 The Surveillance, Epidemiology, and End Results (SEER) population-based program is

256 supported by the National Cancer Institute. Approval from the Institutional Research Board

257 (IRB) was waived due to the data being anonymous and freely accessible.

\section{Consent for publication}

259 Not applicable

\section{Conflict of interest}

261 The authors declare no conflicts of interest.

\section{Availability of data and materials}

263 The data that support the findings of this study are openly available in the SEER database, at

264 http://seer.cancer.gov.

\section{Competing interests}

266 The authors declare that they have no competing interest.

\section{Funding}

268 This research received no specific grant from any funding agency in the public, commercial, 269 or not-for-profit sectors.

\section{Authors' contributions}

271 ZQQ and GZY analyzed and interpreted the patient data regarding the stage IV melanoma,

272 ZQQ was a major contributor in writing the manuscript. XXZ administrated project and

273 reviewed.All authors read and approved the final manuscript.

\section{Acknowledgements}

275 My sincere appreciation goes to the teachers and students from the second hospital of Dalian 276 medical university, who participated this study with great cooperation.

277 Last but not least, I' d like to thank all my friends for their encouragement and support.

\section{References}


1. Jin Y, Siying C, Yuanjie L, et al. Incidence rate and risk factors for suicide death in patients with skin malignant melanoma: a Surveillance, Epidemiology, and End Results analysis. Melanoma Res. 2020;30(4):402-409. doi: 10.1097/cmr.0000000000000559

2. Everett L, Copperman T. Metastatic uveal melanoma. N Engl J Med. 2019;380(19):1853. doi: 10.1056/NEJMicm1810596

3. Howard JH, Thompson JF, Nicola MMD, et al. Metastasectomy for distant metastatic melanoma: analysis of data from the first Multicenter Selective Lymphadenectomy Trial

4. Wevers KP, Hoekstra HJ. Stage IV melanoma: completely resectable patients are scarce. (MSLT-I). Ann Surg Oncol. 2012;19(8):2547-2555. Ann Surg Oncol. 2013;20(7):2352-2356. doi: 10.1245/s10434-013-2881-1

5. Pavri SN, Han G, Khan S, Han D. Does sentinel lymph node status have prognostic

6. Elias ML, Behbahani S, Maddukuri S, John AM, Schwartz RA, Lambert WC. Prolonged overall survival following metastasectomy in stage IV melanoma. J Eur Acad Dermatol Venereol. 2019;33(9):1719-1725. doi: 10.1111/jdv.15667

7. Balch CM, Gershenwald JE, Soong SJ, et al. Final version of 2009 AJCC melanoma staging and classification. J Clin Oncol. 2009;27(36):6199-6206. doi:

$$
10.1200 / \text { jco.2009.23.4799 }
$$

8. Dongxiao Z, Yinjun D, Xiubin S, Shuanghu Y, Jinming Y. Surgery of primary tumor improves the survival of newly diagnosed metastatic melanoma: a population-based, propensity-matched study. Cancer Manage Res. 2018;11:339-346. doi:

\subsection{7/cmar.s187208}

9. Elias ML, John AM, Weisberger JS, Lambert WC, Schwartz RA. Declining recommended surgery in primary cutaneous melanoma: an analysis of risk factors and survival. J Eur Acad Dermatol Venereol. 2019;33(7):e253-e254. doi: 10.1111/jdv.15468

10. Karimkhani C, Gonzalez R, Dellavalle RP. A review of novel therapies for melanoma. Am J Clin Dermatol. 2014;15(4):323-337. doi: 10.1007/s40257-014-0083-7 
11. Smith MJF, Smith HG, Joshi K, et al. The impact of effective systemic therapies on surgery for stage IV melanoma. Eur J Cancer. 2018;103:24-31. doi:

$$
\text { 10.1016/j.ejca.2018.08.008 }
$$

12. Richard T, Camila A, Sharon S, Deutsch GB. Surgical resection of metastatic melanoma in the era of immunotherapy and targeted therapy. Melanoma Manag. 2017;4(1):61-68. doi: $10.2217 / \mathrm{mmt}-2016-0018$

13. Friedman EB, Thompson JF. Continuing and new roles for surgery in the management of patients with stage IV melanoma. Melanoma Manag. 2018;5(1):MMT03. doi:

$$
10.2217 / \mathrm{mmt}-2017-0024
$$

14. Testori AAE, Blankenstein SA, van Akkooi ACJ. Surgery for metastatic melanoma: an evolving concept. Curr Oncol Rep. 2019;21(11):98. doi: 10.1007/s11912-019-0847-6

15. Miller KD, Siegel RL, Lin CC, et al. Cancer treatment and survivorship statistics, 2016. CA Cancer J Clin. 2016;66(4):271-289. doi: 10.3322/caac.21349

16. Korn EL, Liu P-Y, Lee SJ, et al. Meta-analysis of phase II cooperative group trials in metastatic stage IV melanoma to determine progression-free and overall survival benchmarks for future phase II trials. J Clin Oncol. 2008;26(4):527-534. doi: 10.1200/jco.2007.12.7837

17. Wollina U, Brzezinski P. The value of metastasectomy in stage IV cutaneous melanoma. Wien Med Wochenschr. 2019;169(13-14):331-338. doi: 10.1007/s10354-018-0630-6

18. Linette GP, Carlson JA, Slominski A, Mihm Jr MC, Ross JS. Biomarkers in melanoma: stage III and IV disease. Expert Rev Mol Diagn. 2005;5(1):65-74. doi: 10.1586/14737159.5.1.65

19. Phan K, Loya A. Mohs micrographic surgery versus wide local excision for melanoma in situ: analysis of a nationwide database. Int J Dermatol. 2019;58(6):697-702. doi: 10.1111/ijd.14374

20. Sampson JH, Carter JH, Friedman AH, Seigler HF. Demographics, prognosis, and therapy in 702 patients with brain metastases from malignant melanoma. J Neurosurg. 1998;88(1):11-20. doi: 10.3171/jns.1998.88.1.0011 
21. Gershenwald JE, Scolyer RA, Hess KR, et al. Melanoma staging: evidence-based changes in the American Joint Committee on Cancer eighth edition cancer staging manual. CA Cancer J Clin. 2017;67(6):472-492. doi: 10.3322/caac.21409

22. Namikawa K, Aung PP, Gershenwald JE, Milton DR, Prieto VG. Clinical impact of ulceration width, lymphovascular invasion, microscopic satellitosis, perineural invasion, and mitotic rate in patients undergoing sentinel lymph node biopsy for cutaneous melanoma: a retrospective observational study at a comprehensive cancer center. Cancer Med. 2018;7(3):583-593. doi: 10.1002/cam4.1320

23. Frankel TL, Bamboat ZM, Ariyan C, Coit D, Sabel MS, Brady MS. Predicting the development of brain metastases in patients with local/regional melanoma. J Surg Oncol. 2014;109(8):770-774. doi: 10.1002/jso.23574

24. Zakrzewski J, Geraghty LN, Rose AE, et al. Clinical variables and primary tumor characteristics predictive of the development of melanoma brain metastases and postbrain metastases survival. Cancer. 2011;117(8):1711-1720. doi: 10.1002/cncr.25643

25. Balch CM, Soong S-J, Gershenwald JE, et al. Prognostic factors analysis of 17,600 melanoma patients: validation of the American Joint Committee on Cancer Melanoma Staging System. J Clin Oncol. 2001;19(16):3622-3634. doi: 10.1200/JCO.2001.19.16.3622

26. Luen S, Wong SW, Mar V, et al. Primary tumor thickness is a prognostic factor in stage IV melanoma: a retrospective study of primary tumor characteristics. Am J Clin Oncol. 2018;41(1):90-94. doi: 10.1097/coc.0000000000000226

27 Bønnelykke-Behrndtz ML, Schmidt H, Christensen IJ, et al. Prognostic stratification of ulcerated melanoma: not only the extent matters. Am J Clin Pathol. 2014, 142: 845-856. doi: 10.1309/AJCPW56PHGLFTKZC

\section{Figure legends}

Figure 1 Patient enrollment flowchart 
364 Figure 2 Kaplan-Meier curves for A) Overall Survival (OS) and B) Melanoma-specific 365 survival (MSS) according to surgical choice

366

367 Figure 3 Kaplan-Meier survival curves of the four groups (the $\mathrm{R}_{0}$ group, the primary tumor 368 resection group, the metastasectomy group, and the no-resection group) in the A) non369 ulcerated melanoma cases and B) ulcerated melanoma cases 
Participants were extracted from SEER Research Database version 8.3.6.1 (Nov $2019 \mathrm{Sub}$ ) which maintained by the National Cancer Institute

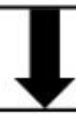

Inclusion criteria:

1. From 2004 to 2015

2. The patients with skin malignant melanoma (Site recode ICD-O-3/WHO 2008)

3. Stage IV using AJCC Stage Group, 6th ed (2004)

4. The patients died of any cause

$\mathrm{N}=8612$
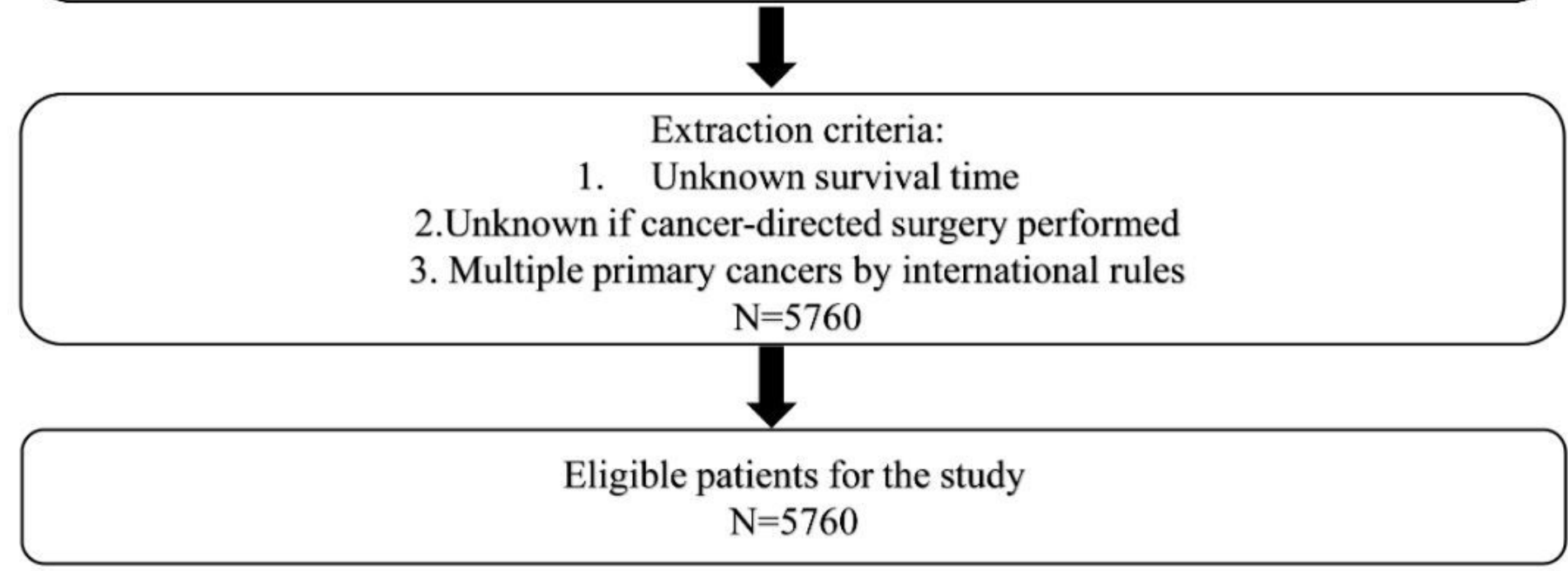

Figure 1

Patient enrollment flowchart
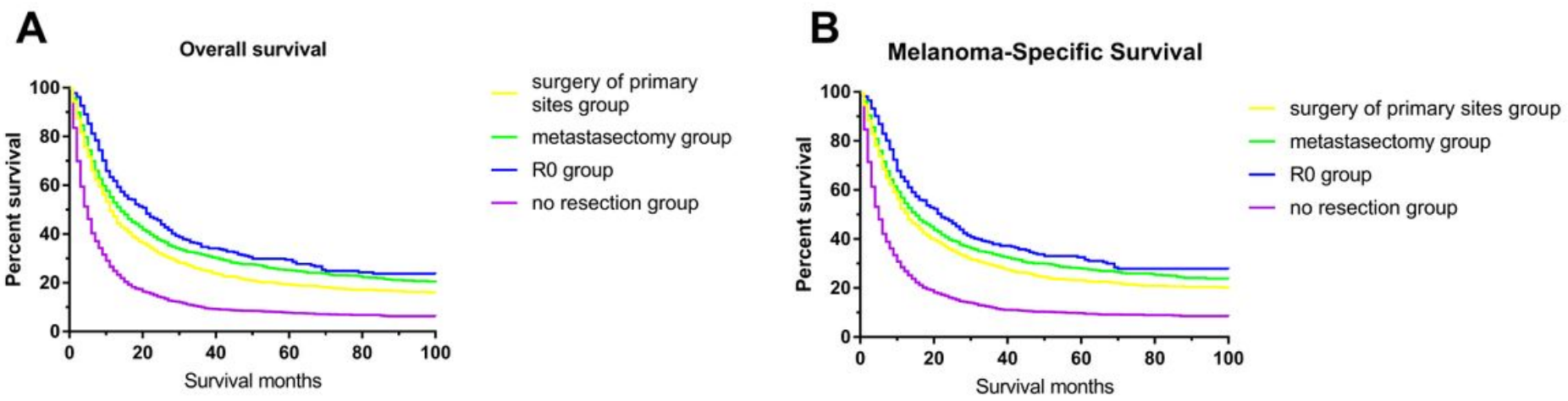

Figure 2 
Kaplan-Meier curves for A) Overall Survival (OS) and B) Melanoma-specific survival (MSS) according to surgical choice
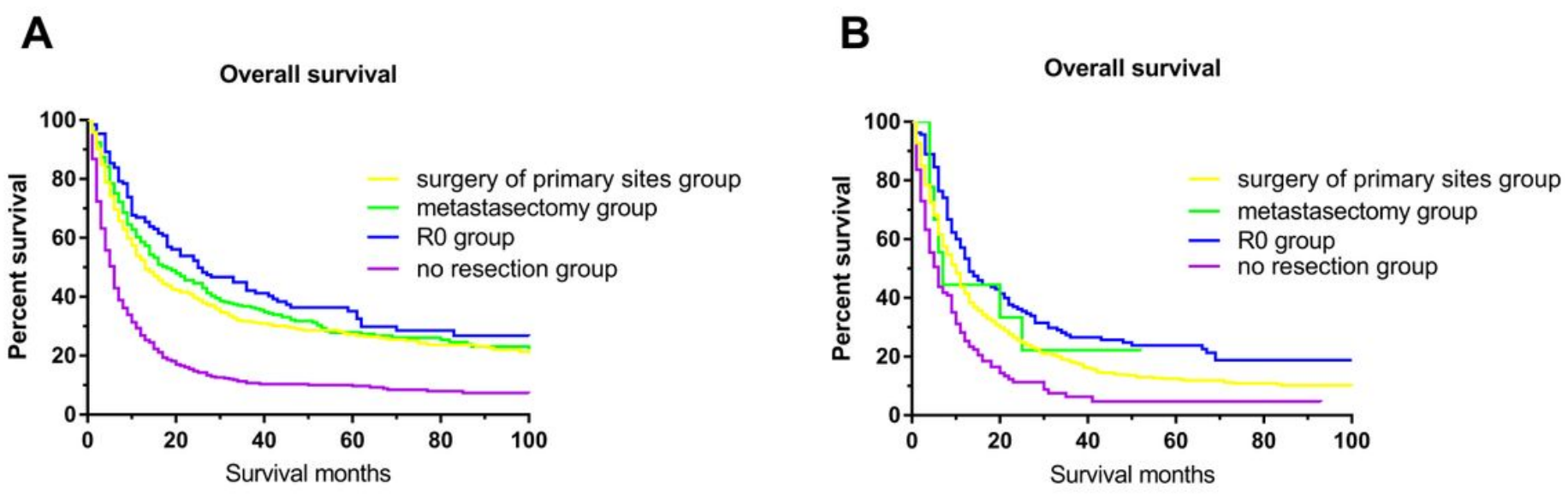

\section{Figure 3}

Kaplan-Meier survival curves of the four groups (the R0 group, the primary tumor resection group, the metastasectomy group, and the no-resection group) in the A) non-ulcerated melanoma cases and B) ulcerated melanoma cases

\section{Supplementary Files}

This is a list of supplementary files associated with this preprint. Click to download.

- Table1.xlsx

- Table2.xlsx

- Table3.xlsx

- Table4.xIsx

- supplement5.xlsx

- supplement6.xlsx 